\title{
Enhanced Magneto-Optical Properties of Suspensions of Spindle Type Mono-Dispersed Hematite Nano-Particles in Liquid Crystal
}

\author{
E. OUSKOVA, ${ }^{1}$ O. BULUY, ${ }^{1}$ C. BLANC, ${ }^{2}$ H. DIETSCH, ${ }^{3}$ \\ AND A. MERTELJ ${ }^{4}$ \\ ${ }^{1}$ Institute of Physics, National Academy of Sciences of Ukraine, \\ Kiev, Ukraine \\ ${ }^{2}$ Laboratoire des Colloides, des Verres et des Nano-matériaux (UMR \\ 5587) Université Montpellier II - CC26, France \\ ${ }^{3}$ Adolphe Merkle Institute and Fribourg Center for Nanomaterials, \\ University of Fribourg, Switzerland \\ ${ }^{4}$ Complex Matter Department, J. Stefan Institute, Ljubljana, Slovenia
}

\begin{abstract}
We explored how to increase liquid crystal sensitivity to external magnetic fields. Suspensions consisting of a mixture of liquid crystal and spindle type monodispersed hematite nano-particles at concentrations lower than $1 \mathrm{wt} \%$ were prepared. The Fréedericksz transition threshold for the suspensions appeared to be lower than for the pure liquid crystal. It was proved that adding canted antiferromagnetic nano-particles in liquid crystals increased their sensitivity to magnetic field while no change of the basic mesogenic properties of the matrix occurred.
\end{abstract}

Keywords Liquid crystal; magnetic suspension; spindle type hematite particles

\section{Introduction}

One of the main interests of conventional liquid crystalline media is the possibility to use them in various types of optical devices (optoelectronic, photonic, etc.) and to control their properties under external fields. Usually, this control is realized by electric fields but, in principle, can also be induced by magnetic ones. This would avoid many technical problems and drawbacks caused by the unavoidable presence of charges in liquid crystal (LC) material. The magnitude of coupling, however, between a magnetic field and a conventional LC appeared to be very low making the practical use of these effects difficult. It has long been predicted that low magnetic sensitivity problem could be overcome in suspensions of ferromagnetic particles dispersed in a nematic LC [1], where a magnetic field of the order of 10 Oersteds (compared with $10^{3}$ Oersteds for pure nematics) will be enough to produce profound effects. The possibility of using magnetic rather than electrical

Address correspondence to E. Ouskova, Institute of Physics, National Academy of Sciences of Ukraine, Pr. Nauki 46, Kiev 03028, Ukraine. Tel.: +38044 525 0779; E-mail: ouskova@ iop.kiev.ua 
switching in liquid crystals thus gives future for new types of devices for display or light modulation, or just for magnetic field visualization. A number of attempts were made to find out the predicted reorientation effects [2-4] in magnetic suspensions. The first observation of the uniform molecular orientational distortion of the entire matrix upon the application of the external magnetic fields lower than $1 \mathrm{G}$ in the nematic LC doped with magnetic grains was reported by Chen and Amer [5]. In their system, the composite was produced by placing needles of $\gamma-\mathrm{Fe}_{3} \mathrm{O}_{4}$ with an aspect ratio of 7:1 in the LC matrix. The needles were specially coated to prevent colloidal aggregation, but nevertheless aggregation still occurred. In further work Liang and Chen [6,7] carried out experiments on the magneto-optical effects in magnetic LC composite. It was found that the orientation of suspension of tiny magnetic particles in LC can be notably affected by the magnetic field of the Earth. More recently theoretical works have been carried out $[8,9]$, showing that there was no Fréedericksz transition threshold in such kind of composite, and a strong coupling between the nematic director and the local magnetic moment has been predicted. Recently a more comprehensive theory of ferromagnetic LC colloids was developed [10], presenting that even an ultra-small volume concentration $\left(\sim 10^{-5}\right)$ of anisometric ferromagnetic nano-particles drastically changed the magneto-optics of a nematic cell. Due to the anchoring, the reorientation of the nano-particles in magnetic field resulted in a non-threshold reorientation of LC, and monitoring of magnetic field $\geq 10$ Oe could be easily achieved. At low anchoring between LC and particles' surface, an inverse Fréedericksz effect was predicted, when the nematic reorientation reduced and then disappeared continuously at a critical magnetic field [10,11].

The problem that prevents the real application of magnetic LC composites is their instability due to the particles aggregation occurring after their addition into LC. This problem is not very essential for isotropic liquid hosts, but becomes severe in anisotropic liquid crystal media where additional orientation-elastic forces cause a strong aggregation of the particles.

Taking into account the theoretical prediction $[10,11]$ that even low concentrations of anisometric magnetic particles can affect the magneto-optical properties of LC, and to solve the particles aggregation problem, one can study diluted suspensions of nano-particles in liquid crystals as proposed in [12] for ferroelectric suspensions (which has been realized in Refs. [12-16]). Diluted nano-suspensions are indeed stable, because at those low concentrations the particles would interact weakly. The nano-particles should, however, be small enough in order not to disturb liquid crystal orientation producing macroscopically homogeneous structures. Under such conditions a suspension will appear similar to a pure LC by sight. At the same time, the nano-particles have, however, to be large enough to maintain the intrinsic properties of the bulk material (e.g., ferroelectricity or ferromagnetism), and couple their intrinsic properties with the liquid crystal matrix thanks to the LC anchoring on their surface. The nano-particles size, which is suitable for producing stable suspension, was then estimated to be in the $10^{-8} \div 10^{-6} \mathrm{~m}$ range.

Here we report on an increasing sensitivity of LC to magnetic fields by using a concept of diluted LC suspensions of canted antiferromagnetic nano-particles. Since the coupling of LC molecules with a particle strongly depends upon anchoring anisotropy of the latter, the elongated shape of the introduced nano-sized objects should enhance these effects significantly. This is even more important while dealing 
with magnetic particles because of a rather weak magnetic anisotropy of LC. To increase sensitivity to magnetic fields we therefore used hematite nano-particles with large shape anisotropy of about 5 .

\section{Materials and Experiments}

The magnetic suspensions were prepared by mixing commercial liquid crystal pentyl-cyano-biphenyl (5CB) and spindle type mono-dispersed hematite nanoparticles. Hematite spindle type nano-particles were obtained by forced hydrolysis at $100^{\circ} \mathrm{C}$ of iron III perchlorate $\left(\mathrm{Fe}\left(\mathrm{ClO}_{4}\right)_{3}\right)$ in the presence of urea and sodium dihydrogen phosphate $\left(\mathrm{NaH}_{2} \mathrm{PO}_{4}\right)$ to control the aspect ratio based on previously reported work [17]. $\mathrm{Fe}\left(\mathrm{ClO}_{4}\right)_{3}$ hydrolyses quickly within the first minutes of reaction leading to ellipsoidal paramagnetic particles $\beta-\mathrm{FeOOH}$ also called akaganeite; this intermediate structure is then consumed to precipitate spindle type hematite nano-particles.

The obtained hematite particles $\beta-\mathrm{Fe}_{2} \mathrm{O}_{3}$ are semiconductor $\left(E_{G}=2.1 \mathrm{eV}\right)$ with a high refractive index (3) and high absorption, are chemically stable and non toxic. Hematite nano-particles attracted us by their canted antiferromagnetic properties (Fig. 1) at room temperature, which can be used to test an idea of increasing LC sensitivity to the magnetic field.

Hematite nano-particles were re-dispersed in ethanol at a concentration of $0.63 \mathrm{wt} . \%$. The size and shape of the synthesized nano-particles were checked with SEM (Fig. 2a) by spin-coating a drop of the solution onto a glass substrate, and TEM (Fig. 2b), and proved to be almost mono-dispersed with size about $250 \times 40 \mathrm{~nm}$.

Hematite nano-particles in ethanol were mixed with $\mathrm{LC}$ at room temperature with mechanical mixer during 40-60 min at different concentrations - weight

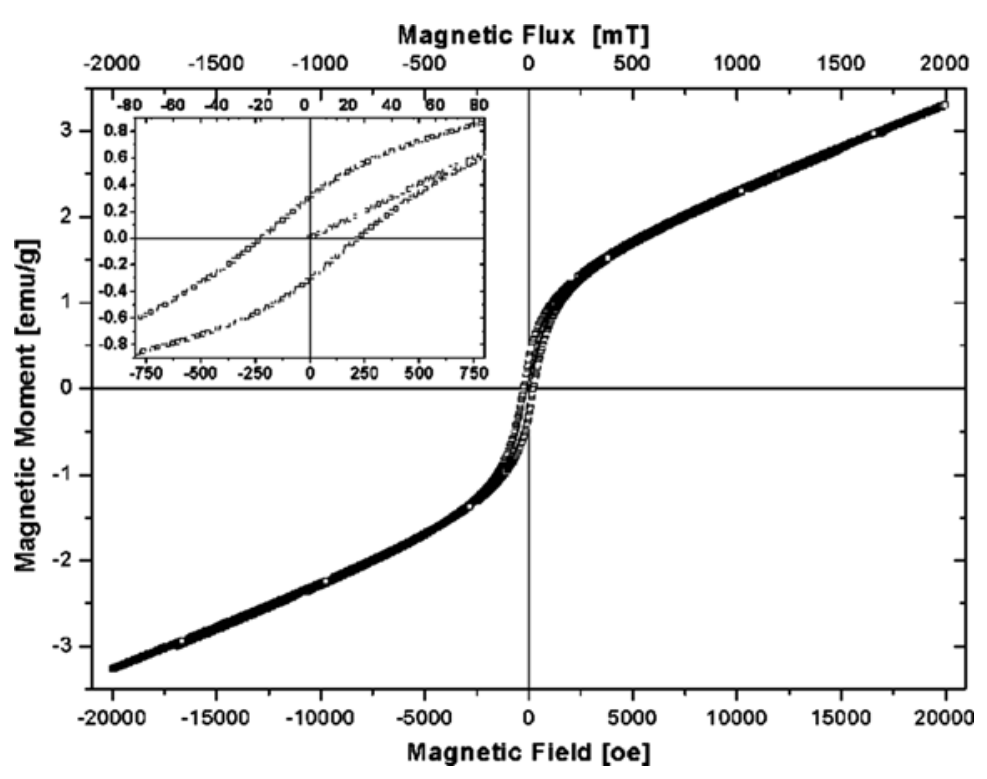

Figure 1. Magnetic properties of hematite nano-particles. 


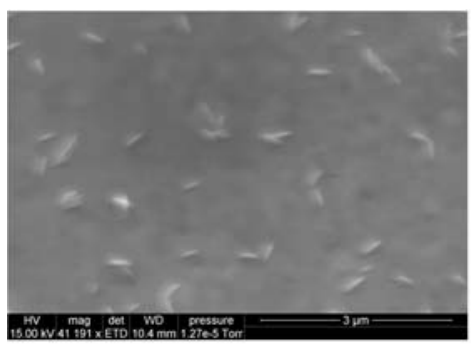

(a)

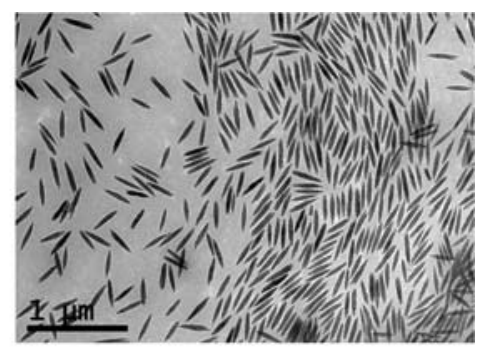

(b)

Figure 2. Observation of hematite nano-particles with SEM (a) and TEM (b) techniques.

fraction $g_{\max }\left(0.014 \mathrm{wt}\left(\mathrm{m}_{\text {particles }} / \mathrm{m}_{\mathrm{LC}}\right), 0.0047,0.0023\right.$, and 0.0012 that is $1.4,0.47$, 0.23 , and $0.12 \mathrm{wt} . \%$ correspondingly. Uniform particles distribution in LC bulk was obtained. The obtained suspensions were put into symmetric LC cells consisted of glass substrates separated by spacer $50 \mu \mathrm{m}$ with planar boundary conditions with strong anchoring provided by rubbed polyimide. The cells were filled with either suspensions or pure LC for comparison.

Optical spectra of magnetic suspensions were measured and compared with pure LC (Fig. 3). The transmittance spectra shape did not changed with increasing concentrations of nano-particles up to $1.4 \mathrm{wt} \%$.

The LC cells were studied in a polarizing microscope, and found to have macroscopically homogeneous planar structures at the lower concentrations of particles in chosen row. At the highest concentration $(1.4 \mathrm{wt} \%$ ) of hematite in LC aggregation processes occurred and aggregates of nano-particles were observed that assumed not using this suspension as diluted one later. The parameters of orientation of the LC suspensions, such as the pretilt angle $\left(2.7^{\circ}\right)$, anchoring energy $\left(10^{-4} \mathrm{~J} / \mathrm{m}^{2}\right)$ were also measured and found to be the same as for the pure LC. The phase transition temperature to isotropic phase $\left(T_{N I}=35.3^{\circ} \mathrm{C}\right)$ also did not change with canted antiferromagnetic nano-particles addition unlike the ferroelectric nano-particles case [16].

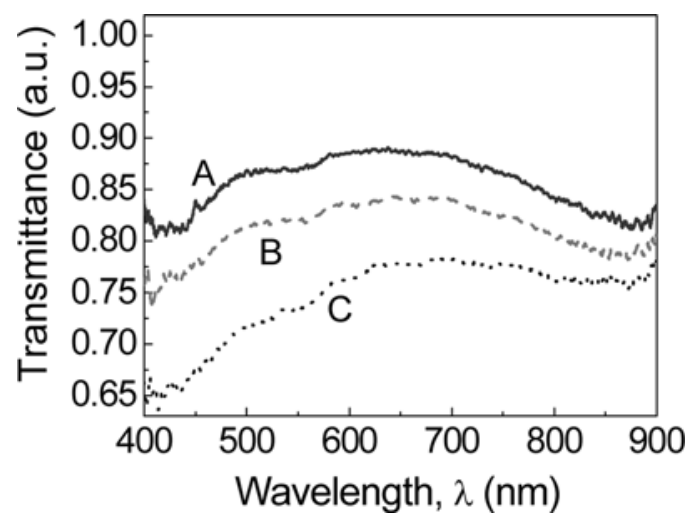

Figure 3. Transmittance spectra of pure LC and suspensions of different concentrations of hematite: A - pure LC; B - LC suspension with $0.47 \mathrm{wt} \%$ of nano-particles; $\mathrm{C}$ - LC suspension with $1.4 \mathrm{wt} \%$ of nano-particles. 
The dynamic light scattering (DLS) experiments were performed to check whether elastic properties (like orientational elastic constants and nematic viscosities) of LC matrix are affected by hematite nano-particles. In the DLS the relaxation times of orientational fluctuation eigenmodes were measured, and they depended on the viscoelastic properties of the nematic LC [18]. The canted antiferromagnetic particles can change viscoelastic properties and can also add new degrees of freedom that result in additional fluctuation modes: similarly as it was observed in suspensions of ferroelectric nano-particles in LC [19].

In our DLS experiment we used a standard photon correlation setup (Fig. 4a) using a frequency-doubled diode-pumped Nd-YAG laser that has excellent power stability and an ALV-6010/160 correlator to obtain the autocorrelation functions of the scattered light intensity. The polarizations of the incoming and scattered light were chosen so that we observed pure bend fluctuation mode (Fig. 4b), that is in the sample with planar orientation and the scattering vector $q$ parallel to the director the bend mode was observed if the incoming light is ordinary polarized and the scattered light extraordinary polarized (o-e scattering).

The measured normalized correlation function $g^{(2)}(q, \tau)=\langle I(q, t+\tau) I(q, t)\rangle /\langle I(q$, $t+\tau)\rangle\langle I(q, t)\rangle$ is related to the field autocorrelation function $g^{(1)}$ by relation $g^{(2)}=1+2\left(1-j_{D}\right) j_{D} g^{(1)}+j_{D}^{2}\left(g^{(1)}\right)^{2}$ with $j_{D}$ being the ratio between the intensity of light, which is scattered inelastically, and the total scattered intensity. In almost all of our measurement the $j_{D}$ was $>0.9$. We fitted our results using an exponential function $\exp (-t / \tau)$ for the field autocorrelation function $g^{(1)}$. The relaxation rate $1 / \tau$ for pure bend mode is $1 / \tau=\left(K_{3} / \eta_{\text {bend }}\right) q_{\|}^{2}$ [18], where $K_{3}$ is bend elastic constant and $\eta_{\text {bend }}$ - the effective bend rotational viscosity.

The DLS measurements were made both for pure LC and magnetic suspensions of different hematite nano-particles concentrations. Figure 5 shows the typical dependence of the relaxation rate on the square of the scattering vector for the pure LC and the suspensions of hematite nano-particles. Linear fitting the data gives the diffusivities $K_{3} / \eta_{\text {bend }}$, i.e., the ratio of the bend elastic constant $K_{3}$ and the effective rotational viscosity $\eta$, for the pure LC and the suspension.

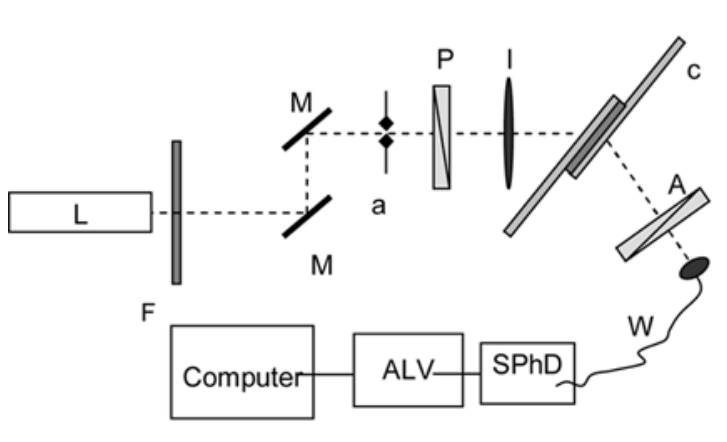

(a)

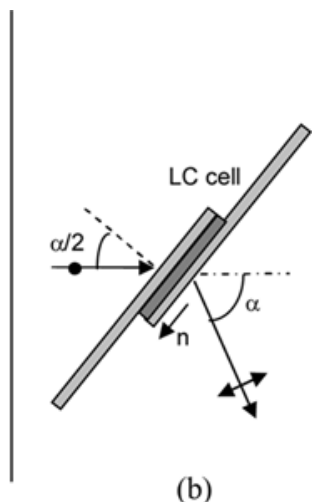

(b)

Figure 4. Setup for Dynamic Light Scattering measurements (a), and experimental geometry (b) for pure bend mode. $\mathrm{L}$ - laser $(\lambda=532 \mathrm{~nm}) ; \mathrm{F}$ - filter; $\mathrm{M}$ - mirror; a - aperture; $\mathrm{P}$ - polarizer; 1 - lens; c - LC cell; A - analyzer; W - wave guide (single mode); SPhD - Single Photon Detector; ALV - Multiple Tau Digital Correlator ALV-6010/160; $\alpha$ - scattering angle, $\mathrm{n}$ - LC director. 


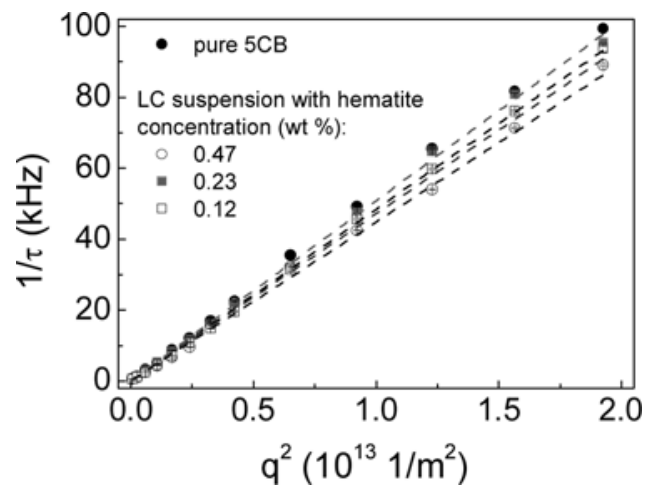

Figure 5. Relaxation rate of the pure bend mode versus the square of the scattering vector $q^{2}$ for pure liquid crystal and LC suspension with different concentration of hematite nano-particles in a planar cell at room temperature. The lines are linear fits to the data.

Within the experimental error the diffusivities are the same for the pure $\mathrm{LC}, 4.84 \pm 0.07 \times 10^{-10} \mathrm{~m}^{2} / \mathrm{s}$, and for the suspensions, for example for $0.47 \mathrm{wt} \%$ of hematite $-4.71 \pm 0.07 \times 10^{-10} \mathrm{~m}^{2} / \mathrm{s}$. Thus, we conclude that the addition of hematite nano-particles in LC matrix does not change the viscoelastic properties of LC.

To see if the canted antiferromagnetic hematite nano-particles added to nematic LC matrix change the magnetic properties, the magneto-optical response was measured. A cell filled either with a suspension or pure LC was placed between crossed polarizers in an electro-magnet providing a magnetic field $H_{\text {test }}$ (Fig. 6). The angle between the LC director and a bias-field $\left(H_{\text {bias }}=0.5 \mathrm{mT}\right)$ direction was

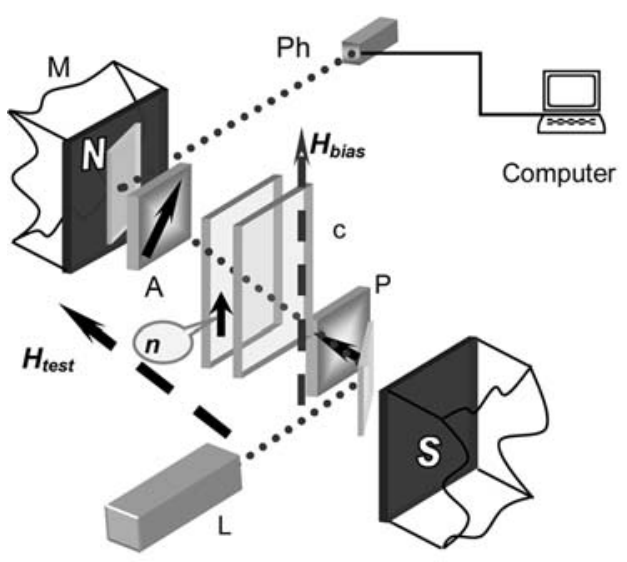

(a)
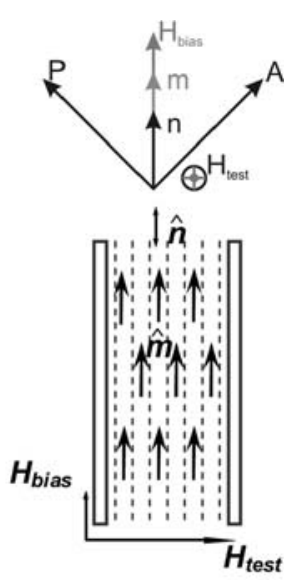

(b)

Figure 6. Experimental setup (a) for measuring magneto-optical response, and geometry of the experiment (b). $\mathrm{M}$ - magnet; $\mathrm{L}$ - laser; $\mathrm{P}$ - polarizer; $\mathrm{A}$ - analyzer; $\mathrm{Ph}$ - photodiode; c - LC cell; $n$ - LC director; $m$ - magnetic moment of the hematite nano-particles; $H_{\text {bias }}-$ magnetic bias-field; $H_{\text {test }}-$ test magnetic field. 


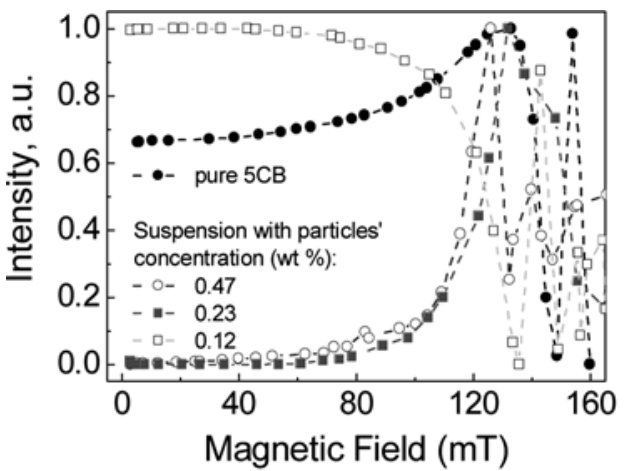

(a)

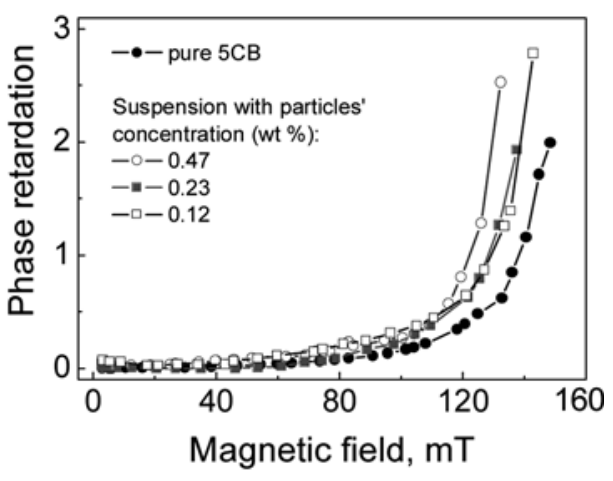

(b)

Figure 7. Dependence of LC and suspensions reorientation (a) and phase retardation in LC cells on magnetic field (b).

$0^{\circ}$. The field $H_{\text {bias }}$ induced additional orientation of the particles, i.e., increased their order parameter in the matrix. The angle between the director and the test-field $H_{\text {test }}$ was $90^{\circ}$. The cells were tested with a laser diode, whose polarization was set at $45^{\circ}$ to director in the cell. The light intensity passed through the LC cell was measured with a photodiode when increasing the magnetic field, and allowed to detect the Fréedericksz transition in magnetic field.

Dependence of the transmitted light intensity on the amplitude of the applied magnetic field is given in Fig. 7a. The corresponding phase retardation in the cells with LC and suspensions has been calculated and its dependence on the magnetic field is presented Fig. $7 b$.

The suspensions revealed an enhanced sensitivity to magnetic field. In particular, our measurements have shown an evident decrease of the Fréedericksz transition threshold in the magnetic field (Fig. 8). It was found that even at small concentrations, $0.47 \mathrm{wt} \%$ of nano-particles, the threshold field decreased for $12 \%$. At higher concentration $\left(1.4 \mathrm{wt}^{\%} \%\right)$ we could achieve the reduction of the threshold field up to $30 \%$, but in that case the suspension could not be treated as a diluted one - there were aggregates of nano-particles in the matrix.

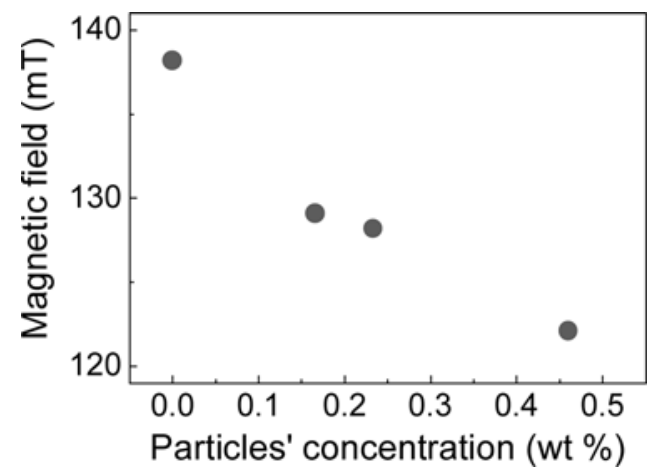

Figure 8. Fréedericksz transition threshold versus hematite nano-particles' concentration. 


\section{Conclusion}

The diluted stable magnetic liquid crystal suspensions consisted of a nematic LC matrix and nano-particles of hematite with spindle type shape were prepared. It was shown that the addition of spindle type hematite nano-particles in a nematic matrix did not change mesogenic, optical, and elastic properties of LC, while the magneto-optical properties of the suspensions were significantly enhanced with increasing nano-particles' concentration, and the Fréedericksz transition threshold for the suspensions appeared to be lower than that for the pure LC. Therefore by producing stable diluted magnetic LC suspension the sensitivity of liquid crystal to magnetic field can be increased noticeably.

\section{Acknowledgments}

The work was done in the frames of the joint Ukrainian-French project (NASU-CNRS) "Ferromagnetic nano-colloids of nematic liquid crystals", and was partially supported by the U.S. Army Research Laboratory and the U.S. Army Research Office under contract number W911NF-08-1-0037, and the Adolphe Merkle Foundation. We are grateful to Yu. Reznikov, M. Čopič, V. Reshetnyak, and A. Iljin for useful discussions.

\section{References}

[1] Brochard, F. \& de Gennes, P. G. (1970). J. Phys. (Paris), 31, 47.

[2] Rault, J., Cladis, P. E., \& Burger, J. P. (1970). Phys. Lett., 32 A, 199.

[3] Hayes, C. F. (1976). Mol. Cryst. Liq. Cryst., 36, 245.

[4] Liébert, L. \& Martinet, A. (1979). J. de Phys. Lett., 40, L-363.

[5] Chen, S.-H. \& Amer, N. M. (1983). Phys. Rev. Lett., 51, 2298.

[6] Chen, S.-H. \& Liang, B. J. (1988). Optics Letters, 13, 716.

[7] Liang, B. J. \& Chen, S.-H. (1989). Phys. Rev. A, 39, 1441.

[8] Burylov, S. V. \& Raikher, Yu. L. (1993). J. Mag. Mag. Mat., 122, 62.

[9] Burylov, S. V. \& Raikher, Yu. L. (1990). Phys. Lett., 149, 279.

[10] Zadorozhnii, V. I., Sluckin, T. J., Reshetnyak, V. Yu., \& Thomas, K. S. (2008). SIAM Jour. Appl. Math., 68(6), 1688.

[11] Zadorozhnii, V. I., Vasilev, A. N., Reshetnyak, V. Yu., Thomas, K. S., \& Sluckin, T. J. (2006). Europhys. Lett., 73(3), 408.

[12] Reznikov, Yu., Buchnev, O., Tereshchenko, O., Reshetnyak, V., Glushchenko, A., \& West, J. (2003). Appl. Phys. Lett., 82(12), 1917.

[13] Ouskova, E., Buchnev, O., Reshetnyak, V., Reznikov, Yu., \& Kresse, H. (2003). Liq. Cryst., 30(10), 1235-1239.

[14] Buchnev, O., Ouskova, E., Reshetnyak, V. Reznikov, Reznikov, Yu., Kresse, H., \& Grabar, A. (2004). Mol. Cryst. Liq. Cryst., 422, 47-55.

[15] Buchnev, O., Cheon, C. I., Glushchenko, A., Reznikov, Yu., \& West, J. L. (2005). Journal of the SID, 13, 749.

[16] Li, F., Buchnev, O., Cheon, C. I., Glushchenko, A., Reshetnyak, V., Reznikov, Yu., Sluckin, T., \& West, J. L. (2006). Phys. Rev. Lett., 97, 1478011.

[17] (a) Ocana, M., Morales, M. P., \& Serna, C. J. (1999). J. Colloid Interface Sci., 12, 317; (b) Dietsch, H., Malik, V., Reufer, M., Dagallier, C., Shalkevich, A., Saric, M., Gibaud, T., Cardinaux, F., Scheffold, F., Stradner, A., \& Schurtenberger, P. (2008). Chimia, 62, 805.

[18] de Gennes, P. G. \& Prost, J. (1993). The Physics of Liquid Crystals, 2 ed., Clarendon Press, Oxford.

[19] Čopič, M., Mertelj, A., Buchnev, O., \& Reznikov, Yu. (2007). Phys. Rev. E, 76, 011702. 